\title{
Scalable FPGA Architectures for LMMSE-based SIMO Chip Equalizer in HSDPA Downlink
}

\author{
Yuanbin Guo, Dennis McCain, Jianzhong Zhang \\ Nokia Research Center \\ 6000 Connection Drive, Irving, TX, USA \\ $\{$ Yuanbin.Guo, Dennis.McCain, \\ Charlie.Zhang\}@nokia.com
}

\author{
Joseph R. Cavallaro \\ Rice University \\ 6100 Main Street, Houston, TX, USA \\ cavallar@rice.edu
}

\begin{abstract}
In this paper, scalable FPGA architectures for the LMMSE-based chip-level equalizer in HSDPA downlink receivers are studied. An FFT-based algorithm is applied to avoid the direct matrix inverse by utilizing the block-Toeplitz structure of the correlation matrix. A PipelinedMultiplexing-Scheduler (PMS) is designed in the front-end to achieve scalable computation of the correlation coefficients. Very efficient VLSI architectures are designed by investigating the multiple level parallelism and pipelining with a Precision-C based High-Level-Synthesis (HLS) design methodology. A 1×2 Single-Input-Multiple-Output (SIMO) downlink receiver is designed and integrated in the HSDPA prototype system with Xilinx Virtex-II XC2V6000 FPGAs. The design demonstrates more area/time efficiency by achieving the best tradeoffs between the usage of functional units and real-time requirements.
\end{abstract}

\section{INTRODUCTION}

With the emerging multimedia services over the wireless cellular systems, the downlink capacity in the $3 \mathrm{G}$ cellular networks is expected to be more crucial than uplink capacity due to the asymmetric data rate requirements. High Speed Downlink Packet Access (HSDPA) is an evolutionary mode for the Wideband CDMA (WCDMA) packet data service for data rate up to $10 \mathrm{Mbps}$ over a $5 \mathrm{MHz}$ bandwidth. The higher data rate is achieved by assigning multiple spreading codes with very short spreading gain to a single user. Although Orthogonal-Variable-Spreading-Factor (OVSF) codes are applied in the HSDPA downlink, the orthogonality of the codes is destroyed by the multi-path fading channel. This results in Multiple-Access-Interference (MAI) at the receiver in addition to the Inter-Symbol-Interference (ISI) caused by the multi-path propagation.

The optimal maximum likelihood multi-user detector was shown to have un-realistic computational complexity that increases exponentially with the number of users. The Rake receiver is shown to provide unacceptable performance in such systems as HSDPA because of the short spreading gain. To achieve a good tradeoff between the performance and complexity, Linear-Minimum-Mean-Square-Error (LMMSE) based chip level equalization has been proposed as one of the most promising sub-optimal receivers $[1,2,3]$. System capacity of the HSDPA system is significantly increased because the orthogonality of the spreading codes is partially restored by the equalization. Recently, receiver diversity techniques using multiple receive antennas or over-sampling are proposed to further increase the system performance.

The equalizer has been one of the most complex blocks in typical communication systems because it usually sets up a problem using the inverse of the correlation matrix, which is very expensive in hardware implementation. The diversity technique makes the design of an equalizer in the downlink even more challenging. To make it feasible for practical implementation, an FFT-based fast algorithm is proposed in [3] by using the banded-Toeplitz structure of the correlation matrix to avoid the matrix inverse and reduce the complexity. The study of efficient VLSI architectures for this algorithm is the focus of this paper, which will be especially interesting for prototyping in a real system and for commercial products.

The complexity of the equalizer is dominant compared with other parts of the HSDPA downlink receiver. There exist many area/time architecture tradeoffs because the algorithm demands different usage of hardware resource and parallelism in different situations. On the other hand, scalability is an important feature to make the design flexible to different channel environments. To achieve the scalability, a PipelinedMultiplexing-Scheduler is designed at the front-end for the computation of correlation coefficients in a streaming mode. A Precision-C based High-Level-Synthesis (HLS) design methodology [4] is then applied to study the multi-level parallelism and pipelining in the algorithm. A 1 transmit $\times 2$ receive antenna SIMO downlink receiver is designed and integrated in the HSDPA prototype system with more area/time efficiency by achieving the best tradeoffs between the usage of functional units and real-time requirements.

\section{SYSTEM MODEL}

In the HSDPA downlink receiver, the chip-level transmitted signal with $K$ active codes is expressed as

$$
d(i)=c(i) \sum_{k=1}^{K} \sum_{m} a_{k} b_{k}(m) s_{k}(i-m G)
$$

where $i$ and $m$ are chip and symbol indices, respectively. Meanwhile, $c(i)$ is the scrambling code, $a_{k}$ is the amplitude, $b_{k}(m)$ is the modulated symbol and $s_{k}(i)$ is the spreading sequence for code $k$. $G$ is the spreading factor of OVSF codes. 




Fig. 1. System diagram of the chip-equalizer.

In a SIMO system where the multi-channel diversity factor is $M$ (note that the multi-channel diversity may be in the form of either over-sampling or multiple receiver antennas), the receiver signal assumes a matrix-vector form of $\mathbf{r}(\mathrm{i})=\mathbf{H d}(\mathrm{i})+\mathbf{n}(\mathrm{i})$, where $\mathbf{r}(\mathrm{i})=[\underline{\mathbf{r}}(i+F), \ldots, \underline{\mathbf{r}}(i), \ldots, \underline{\mathbf{r}}(i-F)]^{\mathrm{T}}$ is the received signal vector of size $M(2 F+1)$ and $\mathbf{d}(i)=[d(i+F), \ldots, d(i), \ldots, d(i-F-L)]$ is the transmitted chip vector of size $2 F+1+L$. For an $L$ path channel, the channel matrix is shown in (2), where each channel tap is an $M \times 1$ vector: $\underline{\mathbf{h}}_{\mathrm{i}}=\left[h_{i, 1}, \ldots, h_{i, M}\right]$.

$$
\mathbf{H}=\left[\begin{array}{lllll}
\underline{\mathbf{h}}_{0} & \cdots & \underline{\mathbf{h}}_{L} & & \\
& \ddots & & \ddots & \\
& & \underline{\mathbf{h}}_{0} & \cdots & \underline{\mathbf{h}}_{L}
\end{array}\right]
$$

An LMMSE chip-level equalizer computes the equalizer taps by minimizing the MSE of the transmitted chip samples and the filtered signal as in $\mathbf{w}^{\text {opt }}=\operatorname{argmin} E\left\|d(i)-\mathbf{w}^{H} \mathbf{r}(i)\right\|^{2}=\mathbf{R}^{-1} \mathbf{h}$, where $\mathbf{h}$ is the $(F+1)^{\text {th }}$ column of $\mathbf{H}$ and $\mathbf{R}=\mathbf{E}\left[\mathbf{r} \mathbf{r}^{H}\right]$ is the correlation matrix. It is shown in [3] that the correlation matrix can be made circulant by adding two corners. FFT operators are applied to make the circulant matrix diagonal and reduce the complexity dramatically. After some elegant numerical operations, it is shown that the equalizer filter taps can be computed with the following approximation,

$$
\mathbf{W}=\mathbf{R}^{-\mathbf{1}} \mathbf{h} \approx\left(\mathbf{D}^{H} \otimes \mathbf{I}\right) \cdot \mathbf{F}^{-1} \cdot(\mathbf{D} \otimes \mathbf{I}) \mathbf{h}
$$

where $\otimes$ denotes the Kronecker product and $\mathbf{D}$ is the DFT matrix. $\mathbf{F}$ is a block-diagonal matrix with elements $\mathbf{F}=$ $\operatorname{diag}\left(\mathbf{F}_{\mathbf{0}}, \mathbf{F}_{\mathbf{1}}, \ldots, \mathbf{F}_{\mathrm{LF}-1}\right)$, which is the element-wise FFT of the first column of a circular matrix. $\mathrm{L}_{\mathrm{F}}$ is the length of the DFT. For the details of the algorithm and performance simulation, please refer to [3].

\section{SCALABLE FPGA ARCHITECTURE}

The focus of this paper is to study the system design and VLSI architectures of the algorithm in [3]. The limited hardware resource and power supply in mobile handsets makes the hardware design more challenging, especially with multiple channel diversity. We emphasize the interaction between architecture, system partitioning and the requirements for the system design flow with three objectives: 1) implement the equalizer with the minimum hardware resource; 2) achieve high-speed update of the equalizer filters; 3) obtain a scalable architecture to accommodate various mobile speeds.

\subsection{Precision-C Design Flow}

As the design progresses from concept to manufacturing, it goes through several levels of abstractions. At each abstraction level, different design views are created in different design representations. In this section, we will present common design representations and examine how they vary from one abstraction level to another. We used the efficient Precision$\mathrm{C}$ based architecture scheduling methodology from Mentor Graphics [4]. The design was conducted using traditional "bottom-up" schematic entry with the Mentor Graphics HDL designer tools. RTL is generated directly from $\mathrm{C} / \mathrm{C}++$ level and imported to the HDL designer for high-level integration. The HDL design is then synthesized using Leonardo Spectrum. Hardware netlist is generated with Xilinx ISE PlaceRoute tools and verified in Nallatech FPGA development platforms.

\subsection{System Level Pipeline}

We illustrate our design blocks for the $M=2$ case, which includes 6 FFT +2 IFFT operations and inverse of $L_{F}$ subblock complex matrices of size $2 \times 2$. The system-level pipeline is designed for a better modularity as in Fig. 1. A module "CompRCC" in the correlation block takes two samples for each chip to compute the correlation matrix $\mathbf{R}_{\mathrm{rr}}=\mathbf{E}\left[\mathbf{r} \mathbf{r}^{\mathrm{H}}\right]$. Then the first column of $\mathbf{R}_{\mathbf{r r}}$ is made circulant by adding a corner to form the matrix $\left[\mathbf{E}_{0}, \mathbf{E}_{1}, \ldots, \mathbf{E}_{\mathrm{L}}, \mathbf{0}, \ldots, \mathbf{0}, \mathbf{E}_{\mathrm{L}}{ }^{\mathrm{H}}, \ldots, \mathbf{E}_{1}{ }^{\mathrm{H}}\right]$, where $\mathbf{E}_{\mathrm{i}}$ is a $2 \times 2$ sub-matrix. The complete coefficients are written to dual-port FPGA RAMs. Four element-wise FFTs compute $\left[\mathbf{F}_{0}, \ldots, \mathbf{F}_{\mathrm{LF}-1}\right]=\mathbf{F F T}\left\{\left[\mathbf{E}_{0}, \ldots, \mathbf{E}_{\mathrm{L}}, \mathbf{0}, \ldots, \mathbf{0}, \mathbf{E}_{\mathrm{L}}{ }^{\mathrm{H}}, \ldots, \mathbf{E}_{1}{ }^{\mathrm{H}}\right]\right\}$. In the meantime, two dimension-wise FFTs are operated on the channel estimates as in $(\mathbf{D} \otimes \mathbf{I}) \mathbf{h}$. A sub-matrix inverse and multiplication block takes the FFT coefficients of both channels and correlations from the DPRAMs and carries out the computation as in $\mathbf{F}^{-1}(\mathbf{D} \otimes \mathbf{I}) \mathbf{h}$. Finally two dimensionwise IFFTs generate the results for the equalizer taps $\mathbf{W}$ and send them to a SIMO FIR block for filtering.

\section{ARCHITECTURE SCHEDULING}

We use Precision-C to do architecture scheduling on different resource/time requirements. From the system level diagram in Fig. 1, we can schedule the tradeoff between latency $T_{i}$ and area $A_{i}$ for each stage to find the most efficient design. Speed is not always linear with the number of FUs because both data dependency and structure hazards stall the pipeline while logic blocks and MUXs determine the clock rate and cycle number. Precision- $\mathrm{C}$ can schedule the order of operations to remove pipeline stalls to some extent. Configurable parallelism is achieved by assigning the number of FUs according to area/time constraints. Multiple ProcessingElement (PE) architectures are generated as in Fig. 2 with configurable parallelism at different levels. The best solution would be the smallest design meeting the real-time requirements. 


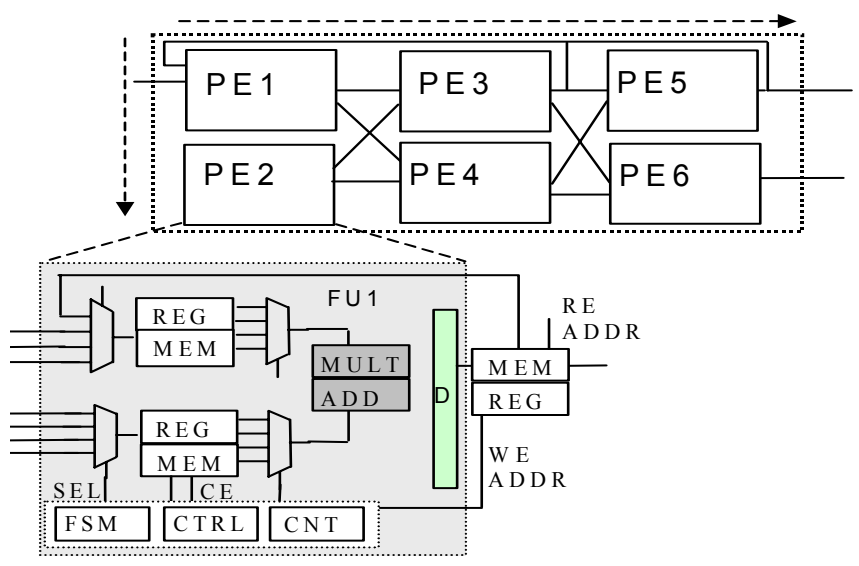

Fig. 2. Precision $\mathrm{C}$ scheduled architecture with multi-level parallelism/pipeline.

In the process of the implementation, we will work on several different modeling levels. Behavioral representation treats the design simply as a black box, while specifying its behavior as a function of its input values and elapsed time. In other words, a behavioral representation describes the system's functionality, but tells us nothing about its implementation. A structural representation begins to answer some of these questions, as it serves to define the black box in terms of a set of components and their connections. It focuses on specifying the product's implementation, and even though the functionality of the black box can be derived from its interconnected components, the structural representation does not describe the functionality explicitly. An architectural representation carries the implementation of the design one step further by specifying the physical characteristics of the components described in the structural representation. Because of the limited space in this paper, we only highlight the important features of some major design blocks.

\subsection{Scalability}

By assuming ergodicity, the ensemble average of the correlation matrix $\mathbf{R}_{\mathbf{r r}}=\mathbf{E}\left[\mathbf{r r}^{\mathrm{H}}\right]$ can be realized by the time average in one block of $N$ samples. The complexity to compute the full correlation matrix is high. However, because of the block Toeplitz structure of the correlation matrix, the correlation matrix is defined by the first block column $\mathbf{E}_{\mathrm{rr}}=\left[\mathbf{E}_{0}, \ldots, \mathbf{E}_{\mathrm{L}}\right]$. Thus the first column block of correlation matrix, i.e., $\mathbf{E}_{\mathrm{rr}}$ is computed as in

$$
\mathbf{E}_{\mathbf{r r}}=\frac{1}{N}\left[\begin{array}{cccc}
\mathbf{r}[0] & \mathbf{r}[1] & \cdots & \mathbf{r}[N-1] \\
& \mathbf{r}[0] & \cdots & \mathbf{r}[N-2] \\
& & & \\
& & \mathbf{r}[0] & \mathbf{r}[N-L]
\end{array}\right]\left[\begin{array}{c}
\mathbf{r}^{H}[0] \\
\mathbf{r}^{H}[1] \\
\vdots \\
\mathbf{r}^{H}[N-1]
\end{array}\right]
$$

where $\mathbf{r}[i]$ is the chip vector that contains $M$ samples at chip index $i$ from all receive antennas. The block size $N$ is determined by the mobile speed. The scalability in the design requires that the design can be flexible to different mobile speeds with no dramatic change of the resource usage.
The independent elements for $\mathbf{E}_{i}(i=1 \sim L)$ are computed as in (5), where $\mathrm{r}_{\mathrm{j}}[\mathrm{n}]$ is the $\mathrm{j}^{\text {th }}$ receiver sample of chip $n$. By composing the algorithm in different structural blocks, we can schedule two different architectures: block mode in Fig. 3 (a) and throughput mode in Fig. 3 (b). Block mode architecture first collects all the $N$ samples in the observation window and then starts computation from the RAM blocks. FSMs for the WE, ADDR/DATA bus and control logic for RAMs are designed to make it work in a similar way as a processor. It has extensive memory access and requires large ping-pong buffers for $N$ samples. Memory stalls will fail the pipeline in computation. The best RTL scheduled from Precision-C has more than $12 \mathrm{~ms}$ latency and could not provide enough parallelism to meet the real-time requirement.

$$
\mathbf{E}_{\mathbf{i}}=\left[\begin{array}{ll}
e_{00}[i] & e_{01}[i] \\
e_{10}[i] & e_{10}[i]
\end{array}\right]=\left[\begin{array}{ll}
\sum_{r_{0}}[n] r_{0}^{*}[n+i] & \sum_{n} r_{0}[n] r_{1}^{*}[n+i] \\
\sum_{n}^{n} r_{1}[n] r_{0}^{*}[n+i] & \sum_{n}^{n} r_{1}[n] r_{0}^{*}[n+i]
\end{array}\right]
$$

To approach real-time computation, a scalable architecture is designed with throughput mode. It completes processing for each sample input within the time resource $\mathrm{F}_{\text {clk }} / \mathrm{F}_{\text {chip }}$ cycles (e.g., 10 cycles for $38.4 \mathrm{MHz}$ clock rate and $3.84 \mathrm{MHz}$ chip rate). With the observation that only the $L$ samples in the observation window are needed in the correlation, $L$ InputShift-Latches (ISL) shift the new samples with the correlated samples in one cycle. The core is the PipelinedMultiplexing-Scheduler (PMS) including a set of FunctionalUnit-Bank (FUB) for both multipliers and adders. The temporary values are stored in Intermediate-MultiplicationRegisters (IMR) and Accumulation-Register (ACR). After a shift operation to adjust the word length, a separate ParallelRead-Shuffle (PRS) module designed by Precision-C reads the registers in parallel for $\left[\mathbf{E}_{0}, \ldots, \mathbf{E}_{\mathrm{L}}\right]$ and writes the memory and shuffles the Hermitian part $\left[\mathbf{E}_{\mathrm{L}}^{\mathrm{H}}, \ldots, \mathbf{E}_{1}{ }^{\mathrm{H}}\right]$. Memory stalls are avoided and scalability is achieved because it can stop at any chip $N$ to adjust to different mobile speed. The latency is only 10 cycles for this architecture.

TABLE. I. STREAMING CORRELATION:

(A). TRADITIONAL LAYOUT WITH SPEED CONTRAINT; (B). AREA/TIME EFFICIENT DESIGN WITH PMS.

\begin{tabular}{c|l|c|c|c|c|c}
\hline \hline \#Cycle & 1 & 2 & 3 & $4-8$ & 9 & 10 \\
\hline \hline MU (a) & 0 & 176 & 0 & 0 & 0 & 0 \\
\hline $\mathrm{AD}(\mathrm{a})$ & 0 & 0 & 136 & 0 & 0 & 0 \\
\hline \hline MU(b) & $\mathbf{0}$ & $\mathbf{2 2}$ & $\mathbf{2 2}$ & $\mathbf{2 2}$ & $\mathbf{2 2}$ & $\mathbf{0}$ \\
\hline AD(b) & $\mathbf{0}$ & $\mathbf{0}$ & $\mathbf{1 7}$ & $\mathbf{1 7}$ & $\mathbf{1 7}$ & $\mathbf{1 7}$ \\
\hline \hline
\end{tabular}

\subsection{Pipelined Multiplexing Scheduler (PMS)}

The computational complexity of the correlation coefficients is determined by the correlation window length $L$. For the throughput mode architecture with streaming input data, the computation must be finished strictly in one chip period. In an example where the correlation window $L=10$, the complexity is 176 multiplications and 136 additions in each chip period. In the PMS, the number of FUs can be assigned ac- 
cording to different time/area constraints. A time-constrained solution can use 176 multipliers and 136 adders all in parallel to complete the computation in 4 cycles. However, the FUs are in IDLE state for 9 cycles. An area-constrained solution will reuse one multiplier and one adder, but has to take more than 176 cycles. The most area-time efficient architecture in 10 cycles is to reuse 22 multipliers and 17 adders. This is shown as the pipelined solution (b) in Tab. I.

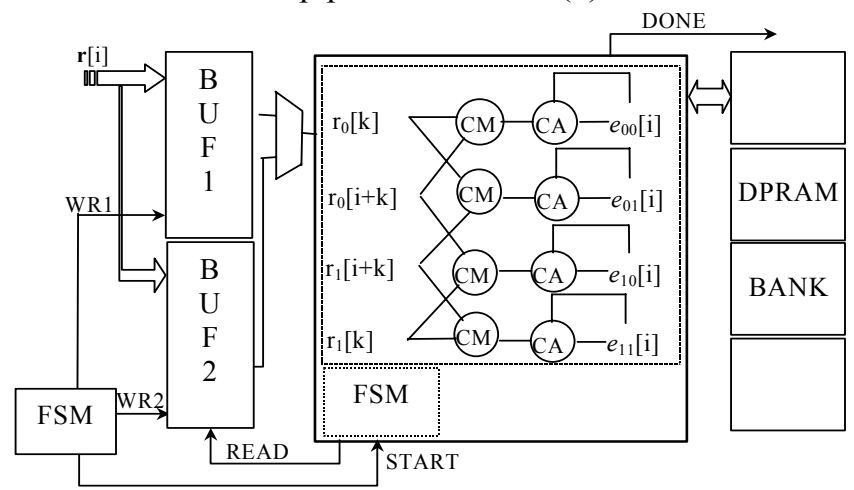

Fig. 3 (a). Block-mode with ping-pong Buffer. CM: complex Multiplier; CA: complex adder.

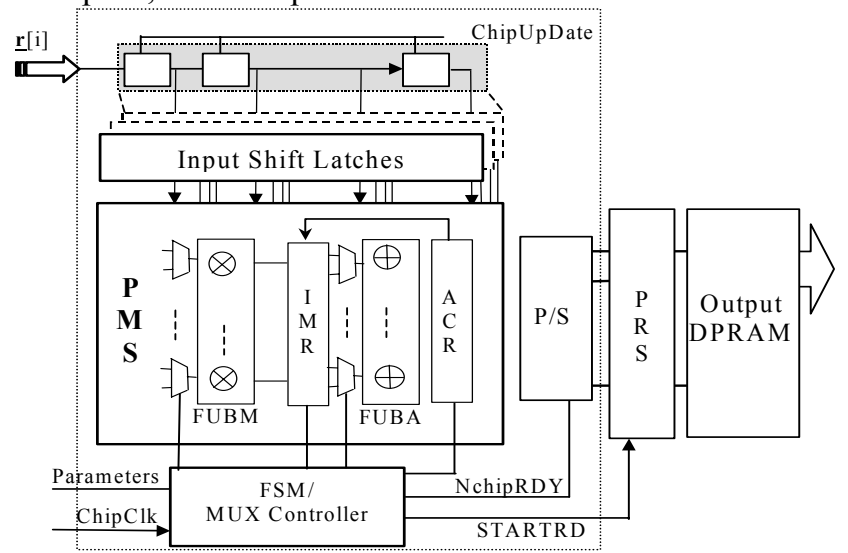

Fig. 3 (b). Scalable throughput mode architecture (FUBM: FUB multipliers; FUBA: FUB adders).

\subsection{Multi-level Pipeline/Parallelism}

Since there are multiple FFTs in the design, the keys for optimization of the area/speed are loop unrolling, pipelining and resource multiplexing. Although Xilinx IP cores for FFTs could be applied for integration, they are much faster than required and the design is considerably large. For example, a single v32FFT core in Xilinx CoreGen library utilizes 12 multipliers and 2066 slices. Since we need 6 FFTs plus 2 IFFTs in one computation period, it is not easy to apply the commonality in the algorithm by using the IP core. We designed the Radix-2 Decimation-In-Time (DIT) FFT algorithm with customized specifications. The initial $\cos / \mathrm{sin}$ phase coefficients for each stage are stored in ROM blocks. Parallelism/pipeline in the parallel FFTs are studied extensively in multi-levels: the Butterfly-Unit (BFU) level, the stage level, and the FFT-processor level. By using MergedButterfly-Unit for M-FFT, we utilize the commonality and different level parallelism and achieve much more efficient resource utilization while still meeting the speed requirement. For a single 32-point FFT with 16 bits precision, the Precision-C scheduled RTL is compared with Xilinx v32FFT Core in Tab. II and demonstrates much smaller size for different solutions, e.g. from solution 1 with 8 multipliers and 535 slices to solution 3 with only one multiplier and 551 slices. Overall, solution 3 represents the smallest design with slower but acceptable speed for a single FFT.

TABLE. II. PRECISION-C 32FFT VS. XILINX V32FFT CORE.

\begin{tabular}{l|c|c|c}
\hline \hline Solution & MULT\# & Cycles & Slices \\
\hline \hline Xilinx v32FFT Core & 12 & 128 & 2066 \\
\hline Precision C Sol_1 & 8 & 570 & 535 \\
\hline Precision C Sol_2 & 2 & 625 & 543 \\
\hline Precision C Sol_3 & $\mathbf{1}$ & $\mathbf{8 1 0}$ & $\mathbf{5 5 1}$ \\
\hline \hline
\end{tabular}

For four element-wise FFTs shown in Fig. 1, we can either lay out duplicate FFT blocks, or just reuse one FFT module in serial computation. In a parallel layout, all the computations are localized and the latency is the same as one single FFT, however, the resource is $4 \times$ of a single FFT module. For a reused module, extra control logic needs to be designed for the multiplexing. The time is equal to or larger than $4 \times$ of the single FFT computation. However, since we merge the FFTs to apply the commonality, we can reuse the control logic inside the FFT module and schedule the number of FUs more efficiently in the integrated mode. The specifications for 4 merged FFTs are listed in Table III with different numbers of multipliers. Compared to 4 parallel FFT blocks (each with 1 MULT) at 2204 slices and 810 cycles or 4 serial-FFT at 3240 cycles, the resource utilization is much more efficient, where FU utilization is defined as: \# Multipliers/(\#Cycles*\#Multiplications).

TABLE III. FOUR MERGED 32-POINT 16-BIT FFT.

\begin{tabular}{c|c|c|c|c}
\hline \hline MULT\# & Slices & Cycles & Util & $\mathrm{F}_{\text {clk }}(\mathrm{MHz})$ \\
\hline \hline 16 & 970 & 570 & $1 / 7$ & 60 \\
\hline 4 & 820 & 810 & $16 / 40$ & 60 \\
\hline 2 & 720 & 1135 & $16 / 28$ & 60 \\
\hline 1 & 680 & 1785 & $16 / 22$ & 60 \\
\hline \hline
\end{tabular}

\subsection{Merged Sub-matrix Inverse and Multiplication}

Another major design block is the matrix inverse and multiplication as in $\mathbf{F}^{-1}(\mathbf{D} \otimes \mathbf{I}) \mathbf{h}$. From equation (3), a straightforward partitioning is at the matrix inversion for $\mathbf{F}$ followed by the matrix multiplication of $\mathbf{F}^{-1}$ and dimension-wise FFT of the channel coefficients. In this partitioning, we would first compute the inverse of the entire sub-block matrix in $\mathbf{F}$ and then carry out a matrix multiplication. However, this partitioning involves two separate the loop structures. Since the two steps have same loop structure, it is more desirable to merge the two steps and reduce the overhead. To have a closer look and derive the expressions for a better partitioning, we expand all the related computations as follows. Because of the diagonal feature of $\mathbf{F}$ matrix, it can be separated into the inverse of $L_{F} \quad 2 \times 2$ sub-matrices as in $\mathbf{F}^{-1}=\operatorname{diag}\left(\mathbf{F}_{0}^{-1}, \mathbf{F}_{1}^{-1}, \cdots, \mathbf{F}_{L_{F}-1}^{-1}\right)$, where 


$$
\begin{aligned}
& \mathbf{F}_{k}^{-1}=\left[\begin{array}{ll}
f_{1,1}(k) & f_{1,2}(k) \\
f_{2,1}(k) & f_{2,2}(k)
\end{array}\right]^{-1} \\
& =\frac{1}{f_{1,1}(k) * f_{2,2}(k)-f_{1,2}(k) * f_{2,1}(k)}\left[\begin{array}{cc}
f_{2,2}(k) & -f_{1,2}(k) \\
-f_{2,1}(k) & f_{1,1}(k)
\end{array}\right]
\end{aligned}
$$

Let $\boldsymbol{\Gamma}=(\mathbf{D} \otimes \mathbf{I}) \mathbf{h}=\left[\begin{array}{llll}\boldsymbol{\Gamma}_{0} & \boldsymbol{\Gamma}_{1} & \cdots & \boldsymbol{\Gamma}_{L_{F}-1}\end{array}\right]^{T}$, where $\boldsymbol{\Gamma}_{k}=\left[\begin{array}{lll}\gamma_{1}(k) & \gamma_{2}(k)\end{array}\right]$ is the combination of the $k^{\text {th }}$ elements of the dimension-wise FFT coefficients, then a merged computation of the matrix inverse and multiplication is given by

$$
\begin{aligned}
& \mathbf{G}=\mathbf{F}^{-1} \cdot(\mathbf{D} \otimes \mathbf{I}) \mathbf{h}= \\
& \operatorname{diag}\left(\mathbf{F}_{0}^{-1}, \mathbf{F}_{1}^{-1}, \cdots, \mathbf{F}_{L_{F}-1}^{-1}\right) \boldsymbol{\Gamma} \\
& =\left[\begin{array}{lllll}
\mathbf{F}_{0}^{-1} \boldsymbol{\Gamma}_{0}^{T} & \mathbf{F}_{1}^{-1} \boldsymbol{\Gamma}_{1}^{T} & \cdots & \mathbf{F}_{L_{F}-1}^{-1} \boldsymbol{\Gamma}_{L_{F}-1}^{T}
\end{array}\right]^{T} .
\end{aligned}
$$

The $k^{\text {th }}$ element in the matrix $\mathbf{G}$ is give by

$$
\begin{array}{r}
\mathbf{W}(k)=\frac{\operatorname{conj}\left[f_{1,1}(k) * f_{2,2}(k)-f_{1,2}(k) * f_{2,1}(k)\right]}{\left|f_{1,1}(k) * f_{2,2}(k)-f_{1,2}(k) * f_{2,1}(k)\right|^{2}} \\
\times\left[\begin{array}{l}
f_{2,2}(k) * \gamma_{1}(k)-f_{1,2}(k) * \gamma_{2}(k) \\
f_{1,1}(k) * \gamma_{2}(k)-f_{2,1}(k) * \gamma_{1}(k)
\end{array}\right] .
\end{array}
$$

Thus we can use a single merged loop to compute the final result of $\mathbf{G}$ instead of using separate loops. The computation data path structure for $\mathbf{W}(\mathrm{k})$ is shown in Fig. 4. In the figure, the multipliers in the boxes denote complex multiplications. For the division, we can first compute the scale using a single real divider instead of using separate dividers as the dotted line indicates. The divider can be made pipelined and be operated concurrently with the multiplication path to reduce the latency. Moreover, the multipliers in the loop can be multiplexed to tradeoff area with speed.

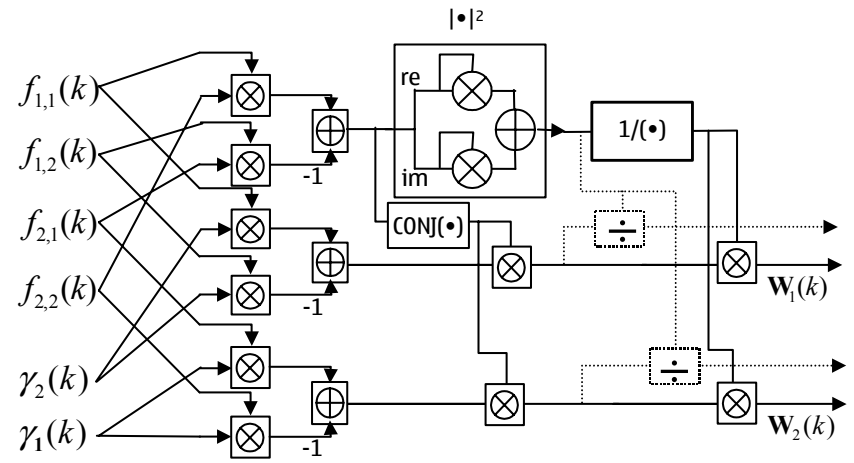

Fig. 4. Data path of the merged $2 \times 2$ sub-matrix inverse and multiplication with dimension-wise FFT output.

\section{PERFORMANCE \& SPECIFICATION}

The overall specifications for FPGA implementation are shown in Table. IV. As the most complex block in the HSDPA receiver, the scalable FPGA implementation consumes about $32 \%$ of Xilinx XC2V6000 CLBs and $22 \%$ of dedicated multipliers. A straightforward implementation is also listed in Italics for comparison. Although a straightforward implementation may consume similar number of slices or CLBs because there is no need to reuse the multipliers with many MUXs, the number of multipliers is dramatically larger than the optimized design with efficient tradeoff analysis. Considering the extensive resource sharing, the overhead in CLB consumption is very efficient. The equalizer coefficients can be updated in less than $100 \mu \mathrm{s}$ at a $38.4 \mathrm{MHz}$ clock rate for 32-tap filters after the correlation coefficients are ready. This hardware prototype has been developed as part of the HSDPA demonstrator in Nokia Research Center, which has been successfully demonstrated in the CTIA 2003 tradeshow. To our knowledge, this implementation is the first working prototype of this technique.

TABLE. IV. SPECIFICATIONS OF THE MAJOR MODULES IN VIRTEX-II XC2V6000 VS. TRADITIONAL LAYOUT DESIGN.

\begin{tabular}{l|c|c|c|c}
\hline \hline Component & \multicolumn{2}{|c|}{ MULT $_{18 \times 18}$} & \multicolumn{2}{c}{ Slices } \\
\hline \hline CompRCC & $\mathbf{2 2}$ & 176 & $\mathbf{6 4 9 6}$ & 4512 \\
\hline InvMUL & $\mathbf{7}$ & 34 & $\mathbf{1 2 9 5}$ & 562 \\
\hline ReadRCC & $\mathbf{0}$ & 0 & $\mathbf{1 1 6 0}$ & 1160 \\
\hline fft32 & $\mathbf{3}$ & 8 & $\mathbf{2 0 4 0}$ & 625 \\
$(\mathrm{X} 8)$ & & $(64)$ & & $(4984)$ \\
\hline Overall & $\mathbf{3 2}$ & 274 & $\mathbf{1 0 9 9 1}$ & 11218 \\
& $\mathbf{( 2 2 . 2 \% )}$ & $(190 \%)$ & $\mathbf{( 3 2 . 5 \% )}$ & $(33 \%)$ \\
\hline \hline
\end{tabular}

\section{CONCLUSION}

We have studied the efficient FPGA implementation for a SIMO chip equalizer in the HSDPA downlink with an architecture scheduling methodology. Several strategies have made the design scalable to channel variation speed and efficient in terms of area/time tradeoffs.

\section{ACKNOWLEDEGEMENTS}

We are grateful to Mentor Graphics for the provision of a Beta license for Precision-C and for the technical support to integrate the complete design flow. We are grateful to Tejas Bhatt for his helpful comments. J. R. Cavallaro was supported in part by NSF under grants ANI-9979465, EIA0224458 and EIA-0321266.

\section{REFERENCES}

[1]. T. P. Krauss, W. J. Hillery, and M. D. Zoltowski, "MMSE equalization for forward link in 3G CDMA: symbol-level versus chip-level", Proc. of 10th IEEE Workshop on Statistical Signal and Array Processing, pp. 18-22, 2000.

[2]. K. Hooli, M. Juntti, M. J. Heikkila, P. Komulainen, M. Latvaaho, J. Lilleberg, "Chip-level channel equalization in WCDMA downlink", EURASIP Journal on Applied Signal Processing, Aug. 2002, pp. 757-770.

[3]. J. Zhang, T. Bhatt, G. Mandyam, "Efficient Linear Equalization for High Data Rate Downlink CDMA Signaling", $37^{\text {th }}$ IEEE Asilomar Conference on Signals, Systems and Computers, 2003.

[4]. Y. Guo, G. Xu, D. McCain, J. R. Cavallaro, "Rapid scheduling of efficient VLSI architectures for next-generation HSDPA wireless system using Precision-C synthesizer", Proc. IEEE Intl. Workshop on Rapid System Prototyping '03, San Diego, CA, pp. 179-185, June2003. 